Anales de Geografía de la Universidad Complutense ISSN: 0211-9803

http://dx.doi.org/10.5209/AGUC.66941

\title{
Urbanismo Subterráneo. Análisis comparativo de las propuestas de Leonardo Da Vinci y Eugène Henard
}

\author{
David Hidalgo García ${ }^{1}$; Julián Arco Díaz ${ }^{2}$; Ana M. Cruz Valdivieso ${ }^{3}$; Juan M. Santiago \\ Zaragoza $^{4}$
}

Recibido: 19 de octubre del 2017 / Enviado a evaluar: 1 de febrero del 2018 / Aceptado: 17 de octubre del 2019

Resumen. Eugène Henard publicó sus Études entre 1902 y 1909. En ellos exponía los problemas urbanos que se estaban desarrollando en la ciudad de París a la vez que ofrecía soluciones de aspecto futurista. Es su propuesta titulada calle subterránea, incluida en la conferencia las ciudades del porvenir, la que hizo que la urbanista Choay lo calificara como "teórico del urbanismo subterráneo". Una propuesta innovadora y única que, sin embargo, presenta múltiples similitudes con la città ideale realizada por el genio del Renacimiento, Leonardo da Vinci cuando se encontraba en Milán entre 14871489. Esta propuesta se encuentra calificada como "un manual para el arquitecto" por los excelentes estudios para la organización de una ciudad de nueva creación que rompía con los sistemas urbanos tradicionales. El artículo realiza una reflexión teórica mediante la comparación entre ambas propuestas que fueron publicadas en distintos ámbitos geográficos y con una diferencia de 422 años. Los resultados arrojan grandes semejanzas y un nexo de unión, la ciudad de París

Palabras clave: Renacimiento; urbanismo subterráneo; città ideale; morfología urbana; París.

\section{[en] Underground Urbanism. A comparative analysis Leonardo Da Vinci and Eugène Hénard}

Abstract. Eugène Hénard published Études between 1902 and 1909. Their pages were numbered as the chapters of a book explaining the problems of the city of Paris and other metropolis of Europe as well as offering futuristic solutions to allow proper functions. Later, he presented documents in conferences and

1 Departamento de Expresión gráfica arquitectónica y en la Ingeniería. Universidad de Granada. E-mail: dhidalgo@ugr.es

2 Departamento de Expresión gráfica arquitectónica y en la Ingeniería. Universidad de Granada. E-mail: juliannn@ugr.es

3 Departamento de Expresión gráfica arquitectónica y en la Ingeniería. Universidad de Granada. E-mail: anacru@ugr.es

4 Departamento de Expresión gráfica arquitectónica y en la Ingeniería. Universidad de Granada. E-mail: santi@ugr.es 
journals filling out the data of previous publications. From his papers -mainly La rue souterraine, included in the conference Les villes de l'avenir-, the town planner Françoise Choay called him the "theorist of the underground urbanism". It was an innovating and unique proposal that had lot of similarities with the città ideale of Leonardo da Vinci. Such a proposal was introduced by this town planner on his Manuscript B, written in Milan between 1487 and 1489, which is considered as an architectural model for organization of a city that breaks away from the traditional urban conceptions. Both authors were studying the underground urbanism, differing 422 years in time. Their only link was the city of Paris.

Key words: Renaissance; underground urbanism; città ideale; urban morphology; Paris.

\section{[fr] Urbanisme Du Sous-Sol. Analyse comparative des propositions de Leonardo Da Vinci et Eugène Henard}

Résumé. Eugène Henard a publié entre 1902 et 1909 ses études, où il exposait les problèmes urbaines qui étaient en train de se développer à la ville de Paris à la fois qu'il donnait des solutions de façon futuriste. Dans sa proposition la rue du sous-sol, inclue à la conférence les citées du futur, laquelle qui fait que l'urbaniste Choay lui qualifier comme théoricien de l'urbanisme du sous-sol. Une proposition innovatrice et unique que, par contre, il présente beaucoup de similitudes avec la città ideale réalisé par le génie de la Renaissance, Leonardo Da Vinci quand il était à Milan entre 1487-1489. Cette proposition est qualifiée comme un manuel pour l'Architect par les excellentes études qu'il propose pour l'organisation d'une ville de nouvelle création qui cassera avec les systèmes urbaines traditionnels. L'article est une pensé théoricien comparative entre les deux propositions publiées dans des différents domaines géographiques avec une différence de 422 ans. Les résultats donnent des grandes similitudes et un lien d'union, la ville de Paris.

Mots clés: Renaissance; urbanisme souterrain; città ideale; morphologie urbaine; Paris.

Cómo citar. Hidalgo García, D., Arco Díaz, J., Cruz Valdivieso, A.M. y Santiago Zaragoza, J.M. (2019): Urbanismo Subterráneo. Análisis comparativo de las propuestas de Leonardo Da Vinci y Eugène Henard. Anales de Geografía de la Universidad Complutense, 39(2), 317-335.

Sumario. 1. Introducción. 2.Marco teórico. 3. Resultados. 4. Discusión. 5. Conclusiones. 6. Bibliografía.

\section{Introducción}

Leonardo da Vinci es considerado como un excepcional artista y genio del Renacimiento y posiblemente de toda la historia de la humanidad. Su actividad profesional más conocida es la de pintor, pero también trabajó como inventor, anatomista, escultor, filósofo y escritor. Menos estudiadas resultan sus aportaciones en los campos de la arquitectura y la morfología urbana, no por ello de menor importancia. Los motivos que justifican esta circunstancia son principalmente dos: la inexistencia de evidencias prácticas de la puesta en marcha de este tipo de trabajos y que nunca cursó estudios oficiales. No obstante, su interés por los problemas y soluciones arquitectónicas y urbanísticas se aprecia en sus Códices y Manuscritos. Posiblemente, de entre todos sus estudios relacionados con la morfología urbana, su propuesta de città ideale con dos niveles de circulación suscite mayor asombro entre 
los estudiosos de la materia y sea considerada como la más fascinante. Fue diseñada tras la epidemia de peste que asoló la ciudad de Milán ${ }^{5}$, durante los años 1484-1485 y presentada a Ludovico Sforza con el objetivo de resolver "infra i popoli pieni d'infiniti mali" (Firpo, 1963: 65). Circunstancia que le ha supuesto ser incluido dentro de la línea de tratadistas renacentistas que estudiaron y propusieron ciudades ideales, entre los que también se incluyen Antonio Averlino "Filarete", Francesco di Giorgio, Vincenzo Scamozzi, etc...

Eugène Hénard (1849-1923), se graduó en arquitectura en la escuela de bellas artes de París en 1880. Aparentemente fue un desconocido dentro del campo del urbanismo ya que sus propuestas se encuentran fragmentadas en múltiples estudios, artículos $^{7}$, conferencias, congresos y no fueron llevadas a la práctica (Evenson, 1971). No obstante, numerosos autores las describen como excepcionales y de una gran inventiva (Benévolo, 2007). Por citar algunos ejemplos: Peter Wolf ${ }^{8}$, lo define como: "Had become the dominant figure in French urban design by the time of the I World War". En 1929, y tras su muerte, el escritor Léandre Vaillat lo describe como: "un precursor [del París moderno]" (Cohen, 2012: 7). También lo cita la arquitecta Françoise Choay como: "el precursor de la tecnotopía" (Choay, 1970: 385), ya que inició la creencia de que los avances en ciencia y tecnología conducirían a una utopía ${ }^{10}$.

La propuesta de Henard conocida como calle subterránea fue el origen de los estudios que comenzaron a desarrollarse en Europa sobre la utilización del subsuelo. Este ya estaba siendo utilizado para la instalación de redes de alcantarillado, abastecimiento, gas y teléfono al objeto de mejorar las condiciones higiénicas de la población pero experimentó un fuerte impulso con la construcción de los túneles

\footnotetext{
${ }^{5}$ Disminuyó en 2/3 la población de la ciudad de Milán, convirtiéndose en un problema político, social y económico. La arquitecta Gaetana Cantone indica: "La pressione demografica, 1 eccesiva densità edilizia, 1 inquinamento dell aria e delle acque, tutte cause di epidemie mortale" (Cantone, 1978: 70), Traducción autor: La presión demográfica, la excesiva densidad urbana y la contaminación del aire y del agua, todas causas de epidemias mortales.

${ }^{6}$ Traducción autor: los pueblos llenos de infinitos males. "Il Pacioli afierma che Leonardo aveva in progetto un trattato sull'architettura, di cui, probabilmente, avrebbero dovuto far parte le note gli schizzi "per una città ideale" contenuti nel Codice B" (Cantone, 1978: 65), Traducción autor: Pacioli indica que Leonardo había planeado un tratado de arquitectura en la que probablemente deberían aparecer los bocetos "para una ciudad ideal", que figuran en el Manuscrito B; Esta circunstancia también es afirmada por el historiador Heydenreich: "Tutta la tematica dell'architettura aveva in mente di ordinare e sistemare nella forma di un vero e proprio tratatto" (Heydenreich, 1972: 39). Traducción autor: Toda la temática arquitectónica, que tenía en mente, ordenada y sistematizada en forma de auténtico tratado.

${ }^{7}$ Antes de publicarlas en folletos, se sirvió de las columnas de las revistas para exponer sus ideas.

${ }^{8}$ Citado en Evenson, 1971: 182.

${ }^{9}$ Traducción autor: Se había convertido en la figura dominante en el diseño urbano francés en la época de la Primera Guerra Mundial.

${ }^{6}$ Esta circunstancia no es compartida por la profesora de pensamiento político Jean Cohen quién defiende que Hénard no fue un utopista, ya que se basa en una postura técnica, no formulando teorías de transformación social (Cohen, 2000).
} 
subterráneos para el metro, sobre todo en las ciudades de Nueva York, Londres y París. En este momento se inició lo que el geógrafo Horacio Capel describe como "la conquista del subsuelo" (Capel, 2005: 501). Este proceso fue posible gracias a las innovaciones técnicas producidas en las máquinas excavadoras y en la instalación de iluminación eléctrica (Capel, 2005).

Si durante los comienzos del siglo XX el uso del subsuelo se planteó como una solución a los problemas ocasionados por el transporte, con el paso del tiempo se abrieron nuevas posibilidades, sobre todo en las urbes ubicadas en climas fríos, tanto para usos comerciales, (Toronto ${ }^{11}$, Montreal y Estocolmo), como para usos deportivos (Gjovic). La angustia desarrollada por la sociedad durante las primeras décadas del siglo XX como consecuencia de los bombardeos sufridos durante las Guerras Mundiales ha permitido también, su estudio desde un punto de vista residencial y de protección (Capel: 2005).

El uso del subsuelo ha sido objeto de investigación por parte de los estudiosos del urbanismo o de la morfología urbana desde los orígenes del siglo XX. No en vano, en 1933 se creó el Grupe d'Etudes et de Coordination de l'urbanisme souterrain en Francia o en 1937 se desarrolló el I Congreso Internacional de Urbanismo Subterráneo (Sorre, 1952). Así, el geógrafo Horacio Capel indica "no extraña que en 1952 Max Sorre [geógrafo francés considerado como uno de los mayores exponentes en el campo de la geografía humana] pudiera escribir "el subsuelo está ya anexionado al espacio urbano" (Capel, 2005: 502). Recientemente el Catedrático de Urbanismo y Ordenación del Territorio, Ángel Martín indico: "La creciente utilización del subsuelo es una realidad asumida con normalidad en la vida cotidiana de las grandes ciudades" (Martín, 2017: 437).

\section{Marco teórico}

El inicio del siglo XX, trajo aparejado el comienzo del tráfico automovilístico, por ello es considerado como el "siglo del automóvil" (Clua, 2017: 65). El aumento indiscriminado de este nuevo tráfico, propició la necesidad de compatibilizarlo con el de peatones y vehículos no motorizados. Circunstancia que fue suficiente para que comenzaran a surgir las primeras teorías del "urbanismo subterráneo" o "del subsuelo" en grandes urbes (Barles y Jardel, 2005: 44). El catedrático de urbanismo Manuel de Solà-Morales utiliza el término "urbanidad subterránea", entendiéndose el término urbanidad como:

\footnotetext{
${ }^{11}$ La ciudad de Toronto cuenta con una red de túneles subterráneos de $30 \mathrm{~km}$. de longitud que alberga tiendas, comercios, museos, oficinas, comercios e incluso un estadio deportivo. Permite a sus ciudadanos realizar actividades sociales y lúdicas cuando las inclemencias del tiempo no son adecuadas. Este conjunto es conocido como Toronto Path.
} 
La urbanidad resulta de la articulación de cosas urbanas, que no depende de las funciones o la actividad, sino de la materia de muros y esquinas, en desniveles y fachadas, en calzadas, aceras, ventanas, portales y vitrinas, en rampas y semáforos, en alineaciones y retranqueos, en gálibos y voladizos, en siluetas y anuncios, en plataformas y vacíos, huecos y descampados.

(Solá-Morales, 2008: 147)

De las primeras décadas del siglo XX, destacan las propuestas de Richard Rummell en 1911 "Future New York"; "Metropolis" de Fritz Lang en 1926; las "ciudades superpuestas" de Lazar Khidekel entre 1922 y 1929; el manifiesto de Édouard Utudjian de 1933 "Urbanisme souterrain" o la propuesta de Le Corbusier de 1951 denominada "Plan de las 7V" (Clua, 2017).

No obstante, es necesario indicar que existen evidencias y propuestas de uso del subsuelo urbano anteriores al siglo XX, sea el caso de las ciudades subterráneas construidas por la civilización Hitita en torno al año 2.000 a.C. en lo que actualmente es la Capadocia (Demir, 1993). Estas presentaban una función de protección de sus habitantes frente a ataques enemigos.

Es necesario dar un salto en el tiempo para nombrar la siguiente propuesta que contempla el uso del subsuelo urbano. Esta es la città ideale realizada por Leonardo da Vinci entre los años 1487-1489 y enmarcada dentro de las propuestas de ciudades ideales de los tratadistas renacentistas. Estas tenían como objetivos romper con la tendencia de desarrollo en las que se inscribían y mejorar las condiciones higiénicas y de salubridad que venía arrastrando la población desde la Edad Media. El autor Evelio Moreno va mucho más allá y afirma: "La città ideale de los renacentistas italianos es la alternativa al paraíso. [. . . ] Este es sustituido por una città como comunidad de vida y pensamiento" (Moreno 1991: 9).

Las teorías higienistas surgidas en los primeros años del siglo XX perseguían ciudades limpias que se alejaran de los desmanes que se estaban produciendo en las urbes con el desarrollo de la Revolución Industrial y el aumento del tráfico automovilístico urbano. La propuesta de calles subterráneas realizada por Henard intenta dar solución a este problema mediante el uso del subsuelo urbano en un momento en el que, como se ha indicado, no se habían iniciado los estudios de ocupación de esta parte de las urbes.

\section{Resultados}

Un análisis detallado de los trabajos realizados por Leonardo da Vinci entre los años 1484-1487 sobre la città ideale y la propuesta de calle subterránea de Eugene Henard realizada entre los años 1902-1909 permite observar las siguientes similitudes:

1. Ambos estudian la ciudad de forma global y proponen soluciones ayudados por los avances en la innovación de la época. Leonardo propone diez ciudades enlazadas 
entre si y divididas en congregaciones: "E trarra 'di dieci città cinquemila case con trentamila abitazioni, e disgregherai tanta congregazione di popolo" ${ }^{\prime 2}$ (Firpo, 1963: 66). Es una ciudad de nueva construcción, sin amurallar, atravesada por un río que se incorpora a la trama urbana ya que permite la circulación de barcas, con dos niveles de circulación, grandes espacios libres para esparcimiento de la población y un trazado con forma de retícula que se divide en congregaciones o "barrios".

Figura 1: Aspecto propuesta città ideale de Leonardo.

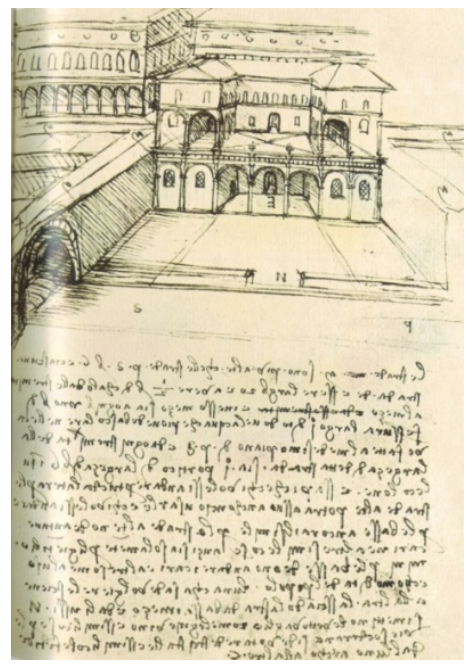

Fuente: Manuscrito B, folio 16r. Biblioteca Ambrosiana da Vinci. Fecha de consulta: Junio 2019.

Una urbe que rompe con el estilo tradicional medieval de núcleo amurallado ${ }^{13} \mathrm{y}$ trazado orgánico que se adelanta cuatrocientos años a las primeras tendencias en este tipo de ciudades (Hidalgo, 2015: 59). De esta forma, la urbe de Leonardo asume un aspecto moderno y burgués (Zollner, 2007: 557) más propio del siglo XIX que del $\mathrm{XV}$, (véase fig. 1), con instalaciones de salubridad, espacios abiertos y accesibles a

\footnotetext{
${ }^{12}$ Traducción autor: Trazar diez ciudades de cinco mil casas con treinta mil habitantes y descomponerla en congregaciones del pueblo.

${ }^{13}$ Las ciudades de la Edad Media eran urbes con una densidad de población muy alta. El motivo de esta circunstancia era la existencia de la muralla de protección que encerraba un espacio limitado pero seguro. Como la ciudad no podía crecer en horizontal, lo hacía en vertical, es decir, en número de alturas de las viviendas. De esta forma se concentraba mayor población por superficie y por tanto la densidad era mayor. Pero este sistema defensivo era un elemento fundamental dentro de una sociedad en lucha por el poder, por lo que su eliminación no era una opción a tener en cuenta.
} 
las innovaciones técnicas de la época ${ }^{14}$. En palabras del historiador Luigi Firpo: "Si tratta di una città signoriale, una giustapposizione di palazzi nobiliari destinati a far corona ad una reggia principesca, o almeno un'accolta di residenze d'una borghesia agiata" $^{15}$ (Firpo, 1963: 80). También, en palabras del historiador Eugenio Garín: "Città spaziosa, ampia, e quindi luminosa, resa viva dall acqua che non serve solo alle comunizacioni sui fiumi, all irrigazione con i canali all igiene, ma quasi ricongiunge alla natura, alla terra e al suo ritmo vitale" ${ }^{" 16}$ (Garín, 1982: 315).

Eugène Hénard estudia la ciudad de una forma global que "le llevará a pensar la capital tanto como una red de equipamientos y como un conglomerado de viviendas" (Cohen, 2012: 16), de tal forma, que no solo estudia la planificación de la urbe de París, sino que expone sus problemas globales de futuro y propone soluciones al respecto. Para él, la ciudad es un todo con diferentes componentes que se enlazan en perfecta armonía, "Buscar sin tregua y sin piedad imperfecciones, incluso las menos visibles, no para tomarlas como objeto de una denigración sistemática, sino para intentar enmendarlas" (Cohen, 2012: 16).

Sus estudios de movilidad y circulación en las ciudades, escritos entre los años 1880 y 1910, "se inscriben en el campo de tensiones entre la innovación y la continuidad de la forma urbana" (Cohen, 2012: 8), en clara alusión a esa capacidad de proponer soluciones adaptadas a las ciudades de comienzos del siglo XX.

2. Establecen un proceso sistemático de estudio que les permite obtener unos resultados. Leonardo utilizó para sus estudios, su propio aprendizaje y el método de la observación, ya que consideraba que todo se encontraba en la naturaleza y por tanto, solo era necesario observarla. Este elemento es importante en Leonardo, ya que la mira de una forma distinta al resto (Mila, 2006: 5). Para él, la observación es una manera de percepción que el filósofo Rudolf Arnheim define como "pensamiento visual" (Arnheim 1971: 13). Es el propio Leonardo quién nos traslada los pasos de su método:

Al abordar un problema científico, dispongo primero diversos experimentos, ya que pretendo determinar el problema de acuerdo con la experiencia, mostrando luego por qué los cuerpos se ven obligados a actuar de ese modo. Ese es el método que hay que seguir en todas las investigaciones sobre los fenómenos de la Naturaleza. Hemos de consultar a la experiencia en

\footnotetext{
${ }^{14}$ Importantes las aportaciones realizadas por la Geógrafa Delfina Trinca en su artículo titulado Territorio y Cambio Tecnológico donde explica como los cambios tecnológicos producidos a lo largo de la historia se han acompañados de cambios en la forma de usar el territorio.

${ }^{15}$ Traducción autor: Se trata de una ciudad señorial, una yuxtaposición de palacios de la nobleza destinados a formar una corona o una región principesca, o al menos un grupo de residencias para una burguesía adinerada.

${ }^{16}$ Traducción autor: Ciudad espaciosa, amplia y, por tanto, luminosa, viva por el agua que no solo sirve para mantenerla comunicada a través de los ríos o para la irrigación mediante canales o para la higiene, sino que integra la naturaleza, la tierra y su ritmo vital.
} 
una diversidad de casos y circunstancias hasta que podamos extraer de ellos una regla general que en ellos se contenga. ¿Para qué son útiles estas reglas? Nos conducen a ulteriores investigaciones sobre la Naturaleza y a las creaciones artísticas. Nos impiden engañarnos a nosotros mismos o a los demás prometiéndonos resultados que no se pueden conseguir.

(Chávez, 2003: 80)

Por su parte, es el propio Henard quién, en una carta a Robert de Souza fechada el 12 de noviembre de 1903, describe su sistema de trabajo: "Antes de escribir uno de mis capítulos, examino un pequeño dosier de notas, de croquis y de recortes que he reunido durante largo tiempo en el transcurso de mis lecturas" (Cohen, 2012: 17). Tras el método descrito, Hénard escribió entre los años 1902 y 1909 sus Études y posteriormente, otras investigaciones que abordan temas concretos y que complementa con ilustraciones que, aunque "resultan en algunos casos esquemáticas, ofrecen, a veces, unas representaciones extraordinariamente detalladas del paisaje de París" (Cohen, 2012: 18).

3. Realizan estudios relacionados con el desarrollo urbano subterráneo en distintos niveles. En la città ideale de Leonardo, las vías de comunicación y los canales están situados en dos niveles: el superior con calles abiertas y soleadas para el paso de personas y, el inferior, oscuro y cerrado, para el tránsito de mercancías a las distintas partes de la ciudad. Se encuentra ubicado bajo los patios de las viviendas y de los establos y está compuesto por vías de tránsito de carros y vías fluviales (Pedretti, 1988). Es el propio Leonardo quien lo confirma:

Por las calles altas no deben pasar carros, ni cosas semejantes, sino solamente los gentiles hombres. Por las calles bajas deberán ir los carros y otras bestias de carga, para uso y comodidad del pueblo. Cada casa deberá dar la espalda a otra, dejando la calle baja en medio.

(Citado en Franchetti, 1985: 570)

En el folio 37v del Manuscrito B (véase fig. 2), se puede apreciar parte de la fachada de un edificio que dispone de planta baja porticada. Los canales se sitúan en el nivel inferior con túneles transversales que permiten la conexión con los almacenes del edificio. Con esta propuesta de circulación en dos niveles, es evidente el objetivo perseguido por Leonardo de garantizar las condiciones de higiene y salubridad de la población en las vías superiores, ya que los desechos de los caballos, obras y mercancías transportadas se quedarían en el nivel inferior, usando el curso fluvial soterrado como gigantesco albañal de evacuación (Hidalgo, 2015: 70). 
Figura 2: Descripción niveles de circulación.

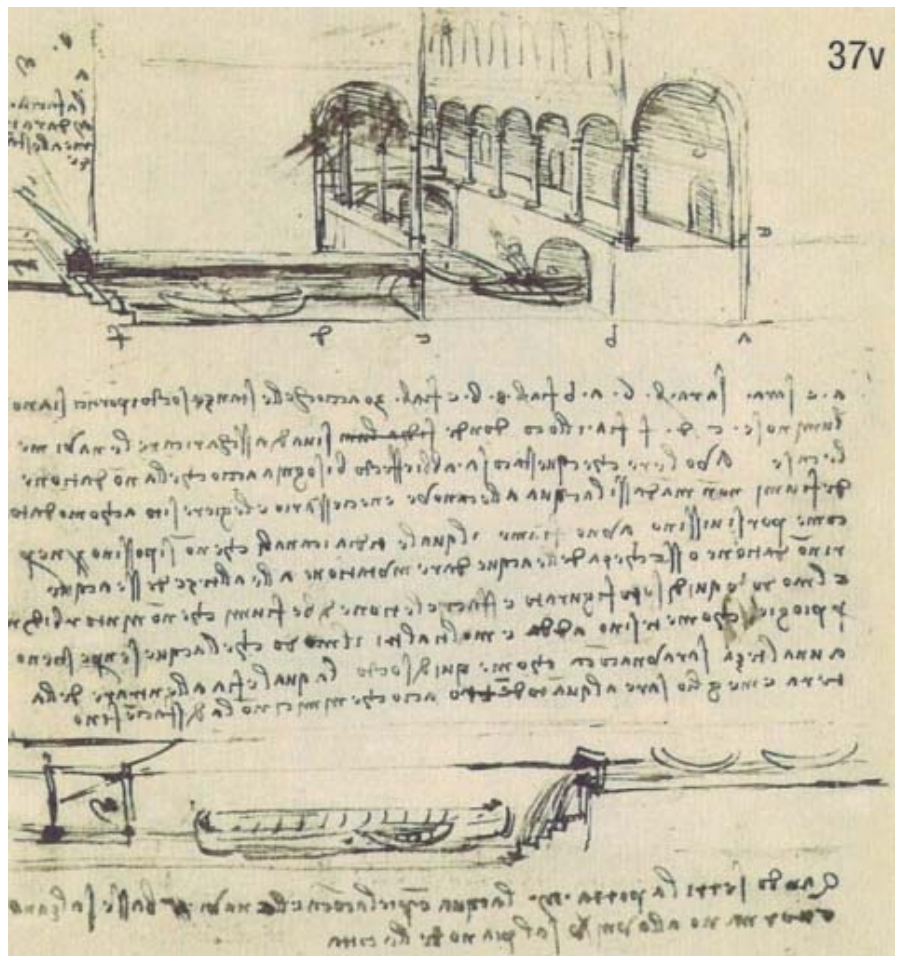

Fuente: Manuscrito B, folio 37v. Biblioteca Ambrosiana da Vinci. Fecha de consulta: Junio 2019.

Leonardo lo confirma con el siguiente comentario: "Per le vie socterane si de' votare destri, stalle e simile cose fetide. Dall' uno arco all'altro de' essere bracci trecento, cioè ciascuna via che riceve il lume dalle fessure delle strade di sopra" ${ }^{17}$ (Firpo, 1963: 75).

Con respecto a la iluminación y ventilación, Leonardo contempló la ubicación de rejillas ubicadas en el suelo del piso superior con el objetivo de permitir la entrada de luz, ventilación y evacuación de aguas de lluvia al nivel inferior.

Tal y como se observa en la figura 3 , la calle del nivel superior presenta una pendiente hacia el centro del vial, lugar donde se ubican las rejillas que permiten la iluminación y la evacuación hacia el nivel inferior. En este último, ocurre lo mismo que en el superior, y la evacuación se realiza mediante rejillas centrales hacia el subsuelo (Fernández-Vivancos, 2016: 142-153).

\footnotetext{
${ }^{17}$ Traducción autor: Por las calles subterráneas inferiores discurra miseria, establos y lugares fétidos similares. De un arco a otro deben ser trescientos brazos, que cada calle reciba la luz de las rendijas de las calles de encima.
} 
Figura 3: Descripción de niveles de circulación.

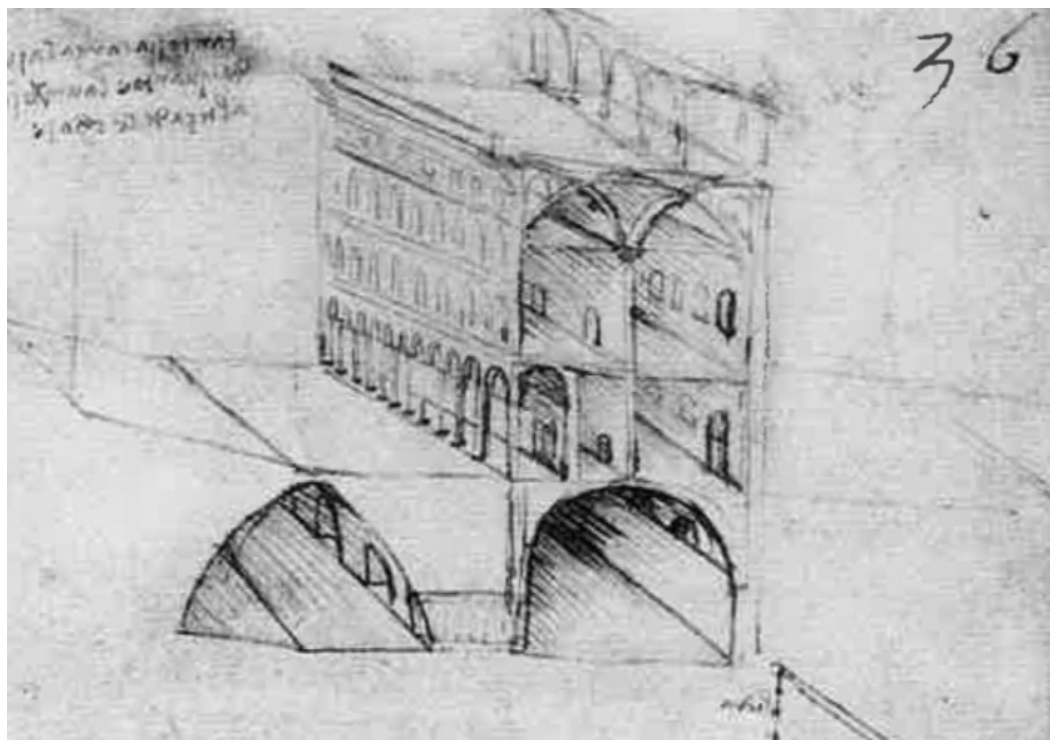

Fuente: Manuscrito B, folio 36r. Biblioteca Ambrosiana da Vinci. Fecha de consulta: Junio 2019.

En relación a la conexión entre ambos niveles de circulación, el artista del Renacimiento ubica estratégicamente en su città una red de escaleras, (véase fig. 4), con las siguientes características: "le scale $\mathrm{c} d$ discendono in $\mathrm{f} g$, e similmente $\mathrm{f} g$ discende in h k" ${ }^{\prime 18}$ (Firpo, 1963: 72).

Figura 4: Elementos de unión entre los niveles de la città.

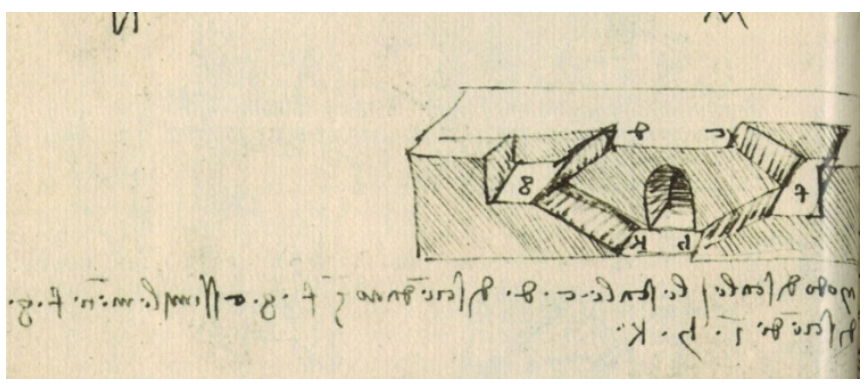

Fuente: Manuscrito B, folio 15v. Biblioteca Ambrosiana Vinci. Fecha de consulta: Junio 2019.

\footnotetext{
${ }^{18}$ Traducción autor: La escalera c y d desciende a f y g y similarmente f y g descienden a h y k.
} 
Esta propuesta ha sido definida por la historiadora Helen Rosenau como "ciudad parcialmente subterránea" (Rosenau, 1986: 62), en clara alusión a la disposición de circulación en dos niveles.

Por su parte, Eugène Hénard publicó en la town planning conference de Londres, celebrada en el año 1910, su propuesta titulada calle subterránea e incluida dentro de la conferencia las ciudades del porvenir. En ella, establece los problemas de las ciudades: "Todos los males provienen de aquella vieja idea tradicional según la cual el suelo de la calle debe establecerse al nivel del suelo natural original" (Hénard, 2012: 305). Se observa como la premisa original de Hénard es romper con esa vieja tradición en el desarrollo urbano de las urbes, por el que la calle se ubica en el nivel del suelo (véase fig. 5), "Puisque tout le mal vient de deux courants intensifs de circulation, supprimons le cause des conflits et faisons passer l'un des courants audessus de láutre ${ }^{19 "}$ (citado en Alonso, 2005: 58).

Figura 5: Planta de calles en París siglo XX.

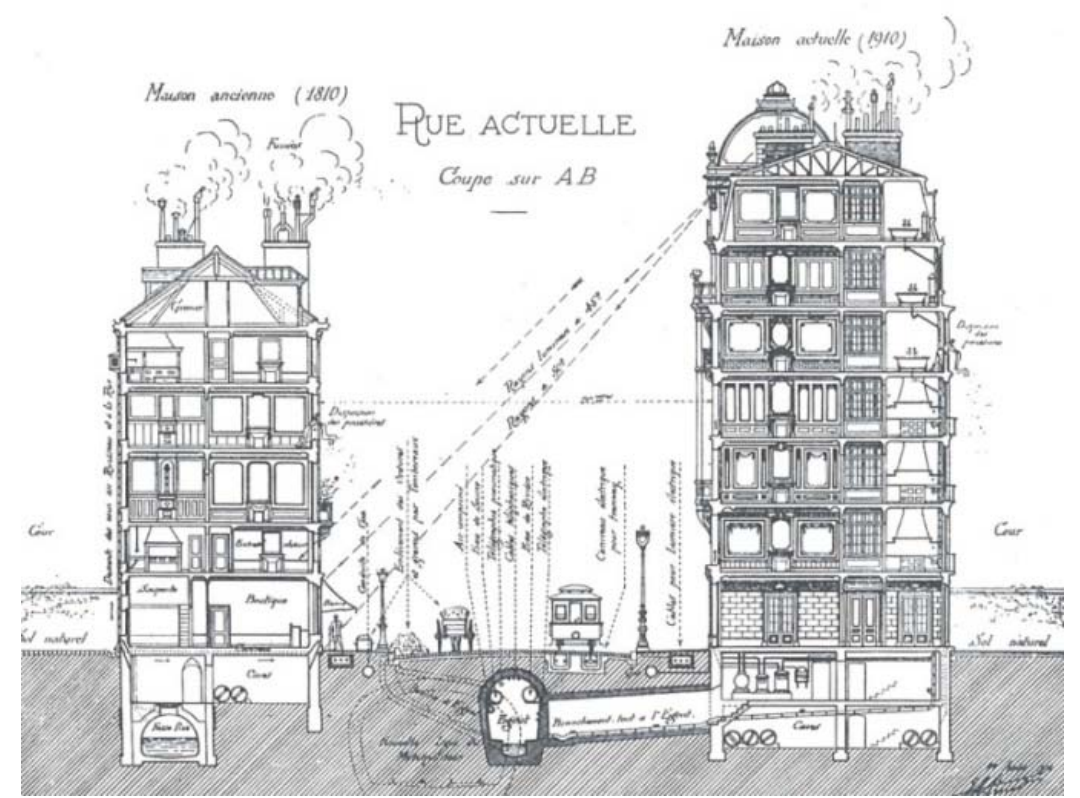

Fuente: Henard, E. (2012): Estudios sobre la transformación de París y otros escritos de urbanismo, pp: 302.

\footnotetext{
${ }^{19}$ Traducción autor: Como todo el mal proviene de dos intensas corrientes de circulación, suprimamos la causa de los conflictos y causemos que una de las corrientes pase sobre la otra.
} 
La idea de usar el espacio del subsuelo tan solo había sido estudiada previamente por Leonardo en el Renacimiento y puesta en marcha en la ciudad de Londres en 1863 con la apertura de la primera línea de metro en la historia, PaddingtonFarringdon Street. París tuvo que esperar hasta el 19 de Julio de 1900 para ver el funcionamiento de la Línea 1 de Metro y todo ello gracias a la electricidad y a las innovaciones en las máquinas excavadoras (Capel: 2005).

Hénard traslada su propuesta subterránea que solucionaría el problema indicado: Pero nada justifica ese error. En efecto, si se parte de la idea contraria según la cual las aceras y la calzada deben establecerse artificialmente a una altura suficiente para dejar por debajo un espacio capaz de acomodar todos los elementos del servicio viario.

(Hénard, 2012: 305)

Por tanto, Hénard establece la necesidad de utilizar el subsuelo de las calles con el objetivo de construir varios niveles de circulación, de tal forma, que el tráfico de mercancías y de basura discurra por los niveles inferiores, mientras que el nivel superior será destinado a tráfico ligero y peatones. Esta propuesta le ha supuesto a Eugène Hénard ser considerado por la urbanista Choay como "teórico del urbanismo subterráneo" (Choay, 1970: 385).

Figura 6: Planta futura de calle con dos niveles de circulación.

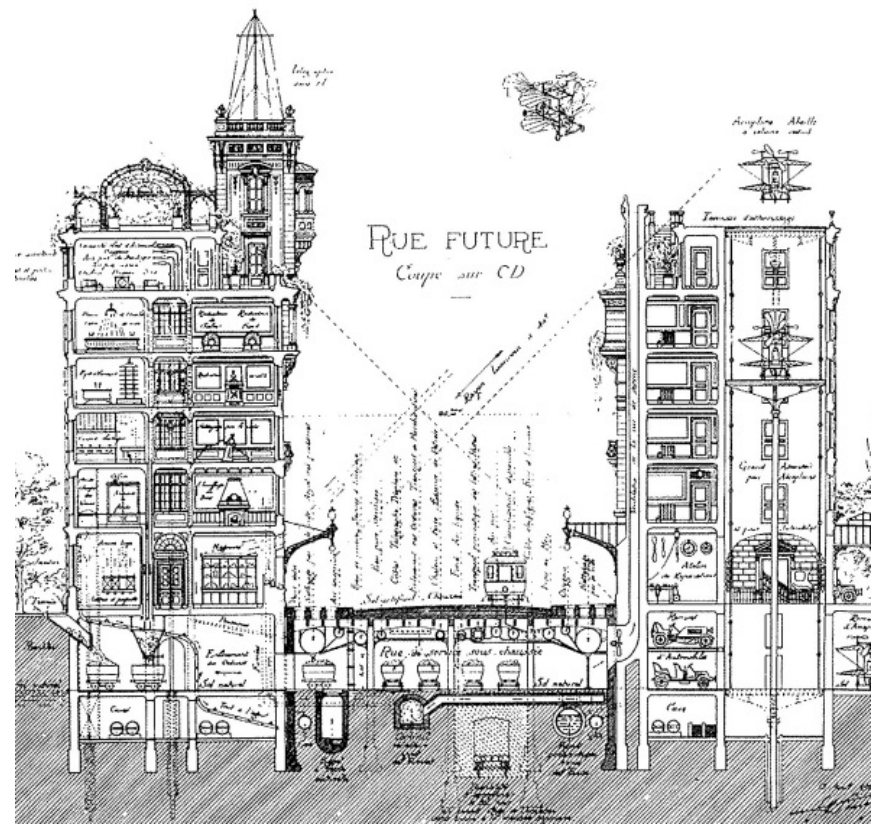

Fuente: Henard, E. (2012): Estudios sobre la transformación de París y otros escritos de urbanismo, pp: 304. 
En la propuesta, (véase fig. 6), se pueden observar dos niveles de circulación: el superior destinado a tráfico ligero y de peatones y el nivel inferior, compuesto por cuatro vías férreas con vagonetas cuya función es la de recoger residuos, basuras generadas en los edificios y transportar materiales pesados y voluminosos. Esta distribución requiere la necesidad de construir un sótano en las viviendas, para así, y mediante plataformas giratorias ubicadas en las naves laterales, permitir la entrada de vagonetas en los sótanos y facilitar la carga y descarga de residuos y su posterior transporte por el nivel inferior.

Por el techo del nivel inferior y con el objetivo de mejorar su mantenimiento, se ubican las canalizaciones de instalaciones: aire comprimido, agua, cableado eléctrico, etc. La altura del nivel inferior será de 2,25 metros. En palabras del propio Hénard:

Un tablero plano que ocupe toda la calle, es mucho más ventajoso, pese a sus múltiples puntos de apoyo, que un túnel con bóveda, porque utiliza todo el espacio disponible. Si incluso la actividad de nuevos organismos exigiese más espacio, o si la creación de una nueva línea de transporte fuese indispensable, se podría excavar más profundamente y despejar el espacio necesario, retomando bajo la obra los puntos de apoyo, y ello con el número cualquiera de plantas subterráneas, sin jamás tocar, trastocar o congestionar la circulación en la vía superior.

(AA.VV, 2012: 306-307)

Por tanto, y observando la descripción realizada por Hénard, se podrían construir tantos niveles de circulación ${ }^{20}$ bajo tierra como fueran necesarios, (véase fig. 7), creando la denominada "calle de plantas múltiples" (Hénard, 2012: 307), que permite la solución de los problemas de circulación de la ciudad de París y del resto de urbes.

En relación a la iluminación y ventilación de los niveles inferiores, Hénard establece que el inferior estará iluminado a lo largo del día mediante "losas de vidrio colocadas al nivel de las aceras" y "lámparas incandescentes" (Henard, 2012: 30). La idea de Henard era que la iluminación fuera tal que apenas se pudiera reconocer estar en el nivel inferior (Clua, 2017: 69). También es tenida en cuenta la ventilación del nivel inferior: "natural, ayudada por ventiladores eléctricos" (Henard, 2012: 306), que expulsarían los gases nocivos a la atmosfera a través de unas altas chimeneas ubicadas en las paredes medianeras de las viviendas, a nivel de fachada.

Con respecto a la comunicación entre los distintos niveles, Hénard establece que ambos niveles deben estar conectados por unas rampas con una pendiente del $5 \%$ y realizadas con armazones de estructura metálica con el objetivo de poder trasladarlas de sitio en función del crecimiento de la ciudad (Henard, 2012: 307). Por tanto, se aprecia como en ambos casos es considerado el elemento de unión entre los distintos niveles de circulación, ya sea mediante escaleras o rampas.

\footnotetext{
${ }^{20}$ Eugéne llega a citar hasta cinco niveles subterráneos, aunque no establece ningún límite.
} 
Figura 7: Calle de plantas múltiples.

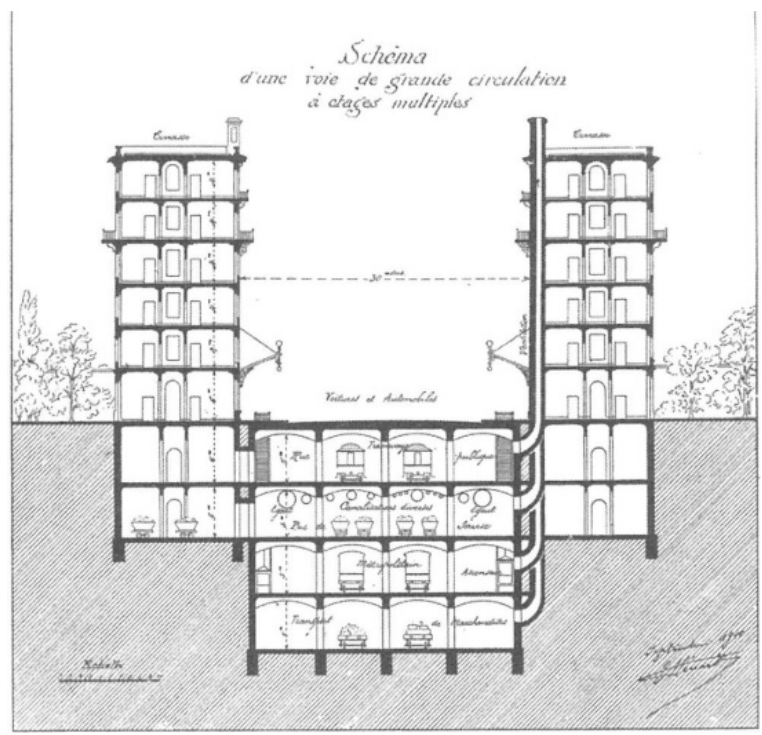

Fuente: Henard, E. (2012): Estudios sobre la transformación de París y otros escritos de urbanismo, pp: 305 .

Por todo lo descrito, se observa como existen similitudes considerables entre las propuestas de ambos autores en relación a la circulación en distintos niveles, usos de estos, conexiones con las edificaciones colindantes, iluminación, ventilación y conexión entre niveles.

El catedrático de urbanismo y ordenación del territorio Ángel Martín califica la propuesta a varios niveles de Hénard como "calle futura" (Martín, 2014: 68), en alusión al carácter práctico y de desarrollo viable que esta solución ha tenido y tendrá en las próximas décadas en todas las urbes. Prueba de ello es la amplia bibliografía que empieza a producirse en este campo a raíz de la propuesta de Henard.

4. Contemplan la ventilación y la salubridad de las viviendas. Leonardo establece la necesidad de ventilación e iluminación en el interior de las viviendas, por lo que las mismas deben contar con ventanas abiertas a las calles. Las dimensiones de estas también se encuentran reguladas, de tal forma que su anchura es igual o mayor a la altura de los edificios (Zollner, 2007). También es necesario disponer de instalaciones de abastecimiento y saneamiento en las viviendas, garantizadas gracias a los canales ubicados a nivel inferior. En la figura 8, correspondiente al folio $47 \mathrm{r}$ del Manuscrito $\mathrm{B}$, se aprecia como el bloque de escaleras del edificio se encuentra en la parte central y Leonardo refleja la distribución de una vivienda (Pedretti, 1988). El resto del edificio es representado mediante líneas esquemáticas debido a la similitud en la 
distribución de todas las viviendas. Destaca de la figura, el ingenioso sistema de escaleras dobles que permiten el acceso a cada vivienda de forma independiente.

Figura 8: Escaleras de edificio en città ideale.

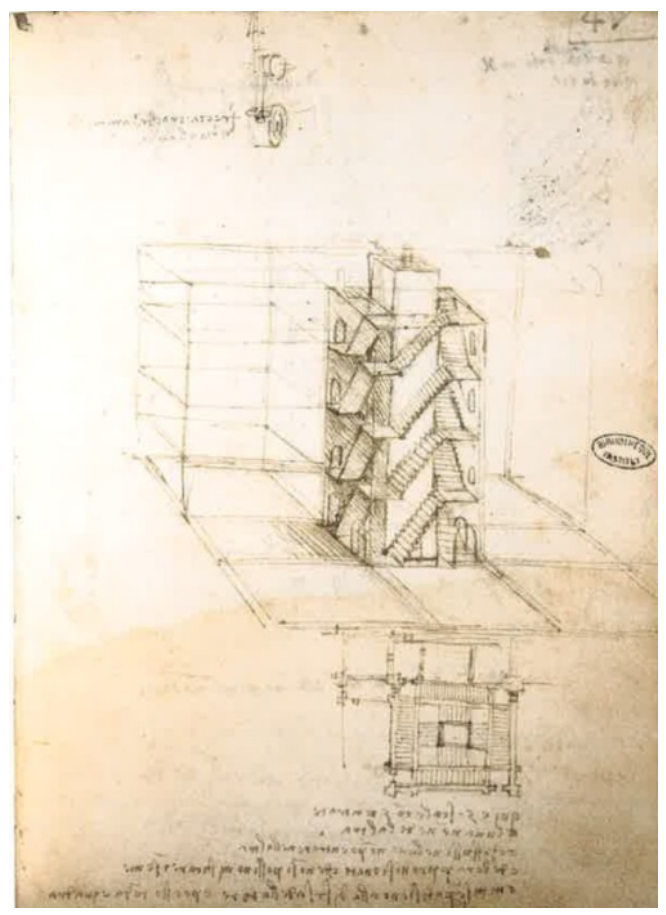

Fuente: Manuscrito B, folio 47r. Biblioteca Ambrosiana da Vinci. Fecha de consulta: Junio 2019.

Tal y como se puede observar en las figuras 6 y 7, Henard también contempla la ventilación y salubridad de las viviendas. Estas disponen de ventanas o balcones a las calles exteriores y para aquellas dependencias que quedan hacia el interior del edificio contempla un patio de ventilación y luz, (véase fig. 6). Es indiscutible que ambos autores profesan una fuerte preocupación por la salubridad e higiene de las viviendas que cuentan con instalaciones de abastecimiento, evacuación de aguas residuales y zona de recepción de mercancías a nivel de sótano.

\section{Discusión}

Se puede indicar que el uso del subsuelo como parte de la vida cotidiana de las personas ha existido desde los orígenes de la civilización (Sabine, 2005). No obstante, no es hasta las primeras décadas del siglo XX y como consecuencia de los avances en 
los sistemas constructivos y medios de locomoción cuando alcanza su momento de mayor esplendor.

A finales del siglo XV el arquitecto y urbanista Leonardo da Vinci reflejó en sus Códices y Manuscritos una propuesta de città ideale que contempla el uso del subsuelo como parte de la urbe. Esta se incluye dentro de las ciudades utópicas del Renacimiento cuyos objetivos eran: defensa militar y corrección de las condiciones de insalubridad que se venían desarrollando en las ciudades de la Edad Media. De esta forma, y durante este periodo comenzaron a aparecer publicados una serie de tratados arquitectónicos y militares que cambiaron el curso de las ciudades, transformándolas de urbes medievales con calles estrechas, tortuosas y sin instalaciones de salubridad en ciudades con espacios abiertos, calles rectas y donde se tenían en cuenta las condiciones de soleamiento, ventilación y salubridad de las viviendas. No obstante, Leonardo fue el único que estudió el uso del subsuelo como parte activa de la ciudad y con el objetivo de mejorar las condiciones higiénicas de la población.

Una de las primeras propuestas del siglo XX de uso del subsuelo como parte de las urbes es la del arquitecto Eugène Henard. Propuesta innovadora que le ha supuesto pasar a la historia del urbanismo como "el precursor" (Cohen, 2012: 7), (Choay, 1970: 385).

Las propuestas de ambos autores distan una diferencia de 400 años aproximadamente pero presentan ciertas similitudes. Ambas estudian problemas globales y proponen soluciones urbanísticas futuras basadas en los avances tecnológicos experimentados en sus periodos. Sus propuestas persiguen mejorar las condiciones de salubridad e higiene de la población y para ello contemplan el uso del subsuelo y mejoras en las instalaciones de ventilación y alcantarillado de las viviendas. Con respecto al uso del subsuelo, varios niveles de circulación permiten desviar el tráfico molesto, insalubre o contaminante hacia los niveles inferiores mientras que el tráfico peatonal discurre por el nivel superior que recibe la radiación solar debido a que la anchura de la calle es igual o mayor que la altura de los edificios. La ventilación de las viviendas se obtiene mediante la apertura de ventanas de grandes dimensiones hacia las calles principales y patios de luces para las dependencias interiores.

Por tanto y aunque resulta evidente que no existen similitudes cronológicasgeográficas entre los dos autores indicados, (Milán 1484 y París 1902), se comprueba que sus propuestas en torno al desarrollo urbano subterráneo son bastante parecidas. ¿Casualidad? La propuesta de città ideale de Leonardo quedó reflejada en el Manuscrito identificado, en el siglo XVIII por el Abad Giovan Battista Venturi, con la letra B (García, 2010). Este fue enviado en 1795 por Napoleón Bonaparte a la Biblioteca del Institut de France en París, donde ha permanecido hasta la actualidad. Fue en esta ciudad en la que Eugene Henard residió y publicó sus estudios entre 1880 y 1910.

El Manuscrito de Leonardo se hizo público en forma de facsímil en 1883, circunstancia que generó un aumento de la admiración hacia el artista por parte de una sociedad en proceso de modernización y cambio en el pensamiento y la cultura. No debe olvidarse que en 1909 aparece en las ciudades de Milán y París el manifiesto 
futurista del escritor Filippo Marinetti, donde propone una síntesis entusiasta de trenes rápidos, aviones y máquinas (Fauchereau, 2006). Con la publicación del facsímil de Leonardo, este pasó a formar parte de la cultura parisina del momento, prueba de ello es la publicación del poeta y filósofo francés, Paul Valéry de 1895, titulada Introduction à la méthode de Léonard de Vinci. En ella, Valéry indica:

Conocía a Leonardo mucho menos de lo que lo admiraba... Por todo esto, el trabajo de su pensamiento pertenece a esa lenta transformación de la noción de espacio, que de una habitación vacía, de un volumen isótropo, se ha convertido poco a poco en un sistema inseparable de la materia que contiene el tiempo.

(Valéry, 1987, pp. 39)

Haciendo clara alusión al asombro que generaban los Códices y Manuscritos de Leonardo sobre el poeta. No obstante, es difícil indicar si el arquitecto Eugène Henard tuvo entre sus manos un facsímil o incluso si pudo consultar el propio Manuscrito B ubicado en la biblioteca del Institut de France en París antes de publicar sus Études pero observando las similitudes de ambas propuestas parece razonable contemplar la hipótesis del conocimiento de la propuesta de Leonardo por parte de Henard.

\section{Conclusiones}

El estudio del subsuelo urbano como parte de las ciudades ha sido un tema de debate entre los estudiosos de la morfología urbana y del urbanismo en las últimas décadas. $\mathrm{Su}$ momento de mayor desarrollo teórico se produjo en los inicios del siglo XX aunque su puesta en práctica no se realizó hasta una década más tarde y como consecuencia del desarrollo de las infraestructuras subterráneas del transporte.

No obstante, a lo largo de la historia de la humanidad han existido propuestas puntuales sobre el estudio del subsuelo urbano. Una de ellas es la propuesta de città ideale de Leonardo da Vinci, considerada como una idea excepcional y de una brillantez majestuosa. Se adelanta 400 años a su época y le otorga el reconocimiento de ser un urbanista excepcional. Así, el historiador Luigi Firpo lo califica como: "è veramente il primo urbanista moderno" 21 (Firpo, 1963: 64), que implica considerar a Leonardo como el promotor del cambio del urbanismo de la época, posibilitando el desarrollo del urbanismo moderno. No es para menos ya que es el único de todos los tratadistas renacentistas que contempla el uso del subsuelo como parte activa de la ciudad. Hay que esperar hasta los inicios del siglo XX para que el arquitecto Eugène Henard publique en sus Études la propuesta de calle subterránea con múltiples niveles de circulación.

\footnotetext{
${ }^{21}$ Traducción autor: Es verdaderamente el primer planificador urbano moderno.
} 
Son apreciables las similitudes existentes entre la propuesta de città ideale de Leonardo da Vinci y la calle subterránea de Eugène Henard, teniendo en cuenta que entre ambas existe una diferencia temporal de aproximadamente 400 años $^{22}$. Durante este periodo de tiempo se produjo una evolución en la tecnología y en las teorías de desarrollo urbano, circunstancia que se hace evidente en la descripción que ambos autores hacen de sus propuestas. No obstante, los principios y características fundamentales de desarrollo de ambas ideas son similares. Se desconocen las teorías o propuestas en las que se basó Henard para el desarrollo de su idea, aunque es posible que conociera el proyecto de Leonardo que se encontraba desde 1795 en la biblioteca del Institut de France en Paris. Esta no cuenta con un registro de lectores del Manuscrito B de Leonardo que nos permita asegurar el conocimiento del contenido del mismo por parte de Henard. No obstante, el Manuscrito B fue publicado en el año 1883 a modo de facsímil, convirtiéndose rápidamente en una publicación popular en París durante varias décadas de finales del siglo XIX.

Con independencia de lo anterior, evidentemente ambas propuestas son magníficas y deben ser consideradas como las precursoras tanto del higienismo como de la "urbanidad subterránea" indicada anteriormente por Manuel de Solà-Morales y que se ha desarrollado durante los siglos XX y XXI.

\section{Bibliografía}

Alonzo, E. (2005): Du rond-point au giratoire. Lyon, Éditions du Certu.

Arnheim, R. (1971): El pensamiento visual. Buenos Aires, Editorial Eudeba, pp. 13.

Evenson, N. (1971): Eugène Henard and the beginning of urbanism in Paris 1900-1914. Journal of the society of architectural historians, volúmen 30, número 2, pp. 180-185.

Benévolo, L. (2007): Historia de la arquitectura moderna. Barcelona. Editorial Gustavo Gili.

Cantone, G. (1978): La cittá di Marmo. Di Alberti a Serlio la storia tra progettazione e restauro. Roma, Oficina edizioni, pp.65-70.

Capel, H. (2005): La morfología de las ciudades: técnica, cultura y clase social en la construcción de edificios. Barcelona, Ediciones del Serbal.

Clua, A. (2017): Urbanidad Subterránea: del Carrefour à étages multiples a la "calle interior". VLC arquitectura. Volumen 4, pp.61-95.

Cohen, J. L. (2012): Eugène Hénard y la modernización de París, en ROJALS, Marta- coord.Estudios sobre la transformación de París y otros escritos de urbanismo, Fundación Caja de Arquitectos, Madrid, pp. 7-18.

Chávez, P. (2003). Comprobación científica: métodos de investigación 2. México, Publicaciones Cultural, pp. 80.

Choay, F. (1970): El urbanismo: utopías y realidades. Barcelona, Editorial Lumne, pp. 385390.

${ }^{22}$ La propuesta de Leonardo es de 1487 y la de Eugene de 1909. 
De Solà-Morales, M. (2008): De cosas urbanas. Barcelona, Editorial Gustavo Gili.

Demir, O. (1993): Capadocia: cuna de la historia. Ankara, Ajans- Türk Matbacilik Sanayi.

Fauchereau, S. (2006): El París de las vanguardias. Lars, número 4, pp. 9-11.

Fernández-Vivancos, E. (2016): Geometría de la transformación. La propuesta urbana de Leonardo da Vinci para Milán. Revista EGA, Volumen 21, número 27, pp. 142-153. http://dx.doi.org/10.4995/ega.2016.4736

Firpo, L. (1963): Leonardo architteto e urbanista. Unione Tipográfico-Editrice Torinese, Torino, pp. 65-80.

Franchetti, V. (1985): Historia del urbanismo: siglos XIV y XV. Traducción de Juan Vioque Lozano. Instituto de Estudios de Administración Local, Madrid, pp. 570.

García, N. (1997): Los Códices de Leonardo en España. Boletín del seminario de Estudios de Arte y Arqueología, Tomo 63, pp. 371-395.

Garín, E. (1972): La cittá in Leonardo. Firenze, Barberá.

Henard, E. (2012): Estudios sobre la transformación de París y otros escritos de urbanismo. Madrid, Fundación caja de arquitectos.

Heydenreich, L. (1943): Leonardo. Berlín, Rembrand verlag.

Hidalgo, D. (2015): La renovación urbana en la ciudad ideal renacentista de Leonardo da Vinci. Arte y Ciudad, núm. 8, pp. 59-82. http://arteyciudad.com/revista/index.php/num1/article/view/214/319

Martín, A. (2014): La calle moderna en 30 autores contemporáneos y un pionero. Barcelona, Universidad politécnica de Cataluña.

Martín, A. (2017): La dimensión oculta de la gran ciudad: Barcelona subterránea. Revista Ciudad y Territorio, estudios territoriales, número 193, volumen XLIX, pp. 437-452. En https://apps.fomento.gob.es/CVP/handlers/pdfhandler.ashx?idpub=BP1021

Mila, M. A. (2006): Leonardo: diseñador del futuro en Conferencias arquitectura y naturaleza. Madrid, Universidad de Alcalá de Henares, pp. 1-8.

Moreno, E. (1991): Las ciudades ideales del siglo XVI. Barcelona, Editorial Sendai.

Pedretti, C. (1988): Leonardo Architetto. Milano, Electa.

Rosenau, H. (1986): La ciudad ideal, la evolución arquitectónica en Europa. Madrid, Alianza, pp. 62.

Sabine, B. \& Jardel, S. (2005). L'urbanisme souterrain:étude comparée exploratoire. Laboratoire Théorie des Mutations Urbaine, Université de Paris.

Sorre, M.(1952): Les fondemets de la géographie humaine. Tome III L`Habitat. Paris, Librairie Armand Colin.

Trinca, D. (2002): Territorio y Cambio Tecnológico. Anales de Geografía de la Universidad Complutense, volumen 22, pp. 67-75.

Valéry, P. (1987). Escritos sobre Leonardo da Vinci. Madrid, Editorial Visor.

Zollner, F. (2007): Leonardo da Vinci: obra pictórica completa y obra gráfica. Traducción de Luis Muñiz. Colonia, Taschen. 